\title{
Hermite-Hadamard Type Inequalities for Multiplicatively $\boldsymbol{P}$-Functions
}

\author{
Çarpımsal P-Fonksiyonlar İçin Hermite-Hadamard Tipli Eşitsizlikler
}

\section{Serap ÖZCAN*}

Department of Mathematics, Faculty of Arts and Sciences, Kırklareli University, Kırklareli, Turkey

• Geliş tarihi / Received: 24.12.2019 • Düzeltilerek geliş tarihi / Received in revised form: $25.02 .2020 \quad$ • Kabul tarihi / Accepted: 06.03.2020

\begin{abstract}
In this study, we first establish some integral inequalities of Hermite-Hadamard type in the setting of multiplicative calculus for multiplicatively $P$-functions. Then, by using some properties of this kind of functions, we obtain new inequalities involving multiplicative integrals for product and quotient of multiplicatively $P$-functions and convex functions.
\end{abstract}

Anahtar kelimeler: Convex Function, Hermite-Hadamard Inequalities, Multiplicative Calculus, Multiplicatively PFunction

$\ddot{\mathbf{O} z}$

Bu çalışmada, öncelikle, çarpımsal P-fonksiyonlar için çarpımsal kalkülüs ortamında Hermite-Hadamard tipi bazı integral eşitsizlikleri oluşturulmuştur. Daha sonra, bu tür fonksiyonların bazı özellikleri kullanilarak, çarpımsal Pfonksiyonlar ile konveks fonksiyonların çarpım ve bölümleri için çarpımsal integralleri içeren yeni eşitsizlikler elde edilmiştir.

Keywords: Konveks Fonksiyon, Hermite-Hadamard Eşitsizlikleri, Çarpımsal Kalkülüs, Çarpımsal P-Fonksiyon 


\section{Introduction and Preliminaries}

The classical or the usual convexity is defined as follows:

The function $\psi:\left[v_{1}, v_{2}\right] \subset \mathbb{R} \rightarrow \mathbb{R}$ is said to be convex if the following inequality holds:

$$
\psi(\lambda x+(1-\lambda) y) \leq \lambda \psi(x)+(1-\lambda) \psi(y)
$$

for all $x, y \in\left[v_{1}, v_{2}\right]$ and $\lambda \in[0,1]$. The function $\psi$ is said to be concave if $-\psi$ is convex.

A number of studies have shown that many of the results obtained about the inequalities are direct consequences of the applications of convex functions. One of the most famous inequalities related to the integral mean of a convex function is Hermite-Hadamard inequality. This double inequality is stated as follows (Dragomir and Pearce, 2000; Pečarić et al., 1992):

Let $\mathfrak{I} \subset \mathbb{R}$ be an interval with $v_{1}, v_{2} \in \mathfrak{J}$ and $v_{1}<v_{2}$ and let $\psi: \mathfrak{I} \rightarrow \mathbb{R}$ be a convex function. Then,

$$
\begin{aligned}
\psi\left(\frac{v_{1}+v_{2}}{2}\right) & \leq \frac{1}{v_{2}-v_{1}} \int_{v_{1}}^{v_{2}} \psi(x) d x \\
& \leq \frac{\psi\left(v_{1}\right)+\psi\left(v_{2}\right)}{2} .
\end{aligned}
$$

In recent years, Hermite-Hadamard integral inequality has attracted the attention of many researchers and a remarkable variety of improvements, generalizations, refinements and applications have been found (Budak et al., 2019; Kadakal, 2018, 2019; Kadakal, 2019; Maden et al., 2018; Özcan, 2019; Özcan and İșcan, 2019; Özdemir et al., 2015; Sarıkaya et al., 2019; Set et al., 2015).

Definition 1.1. (Pečarić et al., 1992) A function $\psi: \mathfrak{J} \rightarrow(0, \infty)$ is said to be multiplicatively or $\log$ convex, if

$\psi((1-\lambda) x+\lambda y)[\psi(x)]^{1-\lambda}[\psi(y)]^{\lambda}$

for all $x, y \in \mathfrak{J}$ and $\lambda \in[0,1]$.

Ali et al. (2019) established Hermite-Hadamard inequality for multiplicatively convex functions as follows:

Theorem 1.2. Let $\psi$ be a positive and multiplicatively convex function on interval $[a, b]$. Then

$$
\begin{aligned}
\psi\left(\frac{v_{1}+v_{2}}{2}\right) & \leq\left(\int_{v_{1}}^{v_{2}}(\psi(x))^{d x}\right)^{\frac{1}{v_{2}-v_{1}}} \\
& \leq G\left(\psi\left(v_{1}\right), \psi\left(v_{2}\right)\right)
\end{aligned}
$$

where $G(.,$.$) is a geometric mean.$

Definition 1.3. (Dragomir and Pearce, 1998) A function $\psi: \mathfrak{J} \rightarrow(0, \infty)$ is said to be quasi convex, if

$\psi((1-\lambda) x+\lambda y) \leq \max \{\psi(x), \psi(y)\}$

for all $x, y \in \mathfrak{J}$ and $\lambda \in[0,1]$.

From the above definitions we have

$$
\begin{aligned}
\psi((1-\lambda) x+\lambda y) & \leq[\psi(x)]^{1-\lambda}[\psi(y)]^{\lambda} \\
& \leq \psi(x)+\lambda[\psi(y)-\psi(x)] \\
& \leq \max \{\psi(x), \psi(y)\}
\end{aligned}
$$

Definition 1.4. (Dragomir et al., 1995) A nonnegative function $\psi: \mathfrak{I} \subseteq \mathbb{R} \rightarrow \mathbb{R}$ is said to be $P$-function if the inequality

$$
\psi((1-\lambda) x+\lambda y) \leq \psi(x)+\psi(y)
$$

holds for all $x, y \in \mathfrak{J}$ and $\lambda \in[0,1]$.

Dragomir et al. (1995) proved the following inequality of Hermite-Hadamard type for $P$ functions:

Theorem 1.5. Let $\psi$ be a $P$-function on $\mathfrak{I}$, $v_{1}, v_{2} \in \mathfrak{I}$ with $v_{1}<v_{2}$ and $\psi \in L\left[v_{1}, v_{2}\right]$. Then

$$
\begin{aligned}
\psi\left(\frac{v_{1}+v_{2}}{2}\right) & \leq \frac{2}{v_{2}-v_{1}} \int_{v_{1}}^{v_{2}} \psi(x) d x \\
& \leq 2\left[\psi\left(v_{1}\right)+\psi\left(v_{2}\right)\right] .
\end{aligned}
$$

Definition 1.6. (Kadakal, 2018) Let $\mathfrak{J} \neq \varnothing$ be an interval in $\mathbb{R}$. A function $\psi: \mathfrak{I} \rightarrow[0, \infty)$ is said to be multiplicatively $P$-function (or $\log -P$ function), if

$\psi((1-\lambda) x+\lambda y) \leq \psi(x) \psi(y)$

holds for all $x, y \in \mathfrak{I}$ and $\lambda \in[0,1]$.

\subsection{Multiplicative Calculus}

Recall that the multiplicative integral is denoted by $\int_{v_{1}}^{v_{2}}(\psi(x))^{d x}$ while the ordinary integral is denoted by $\int_{v_{1}}^{v_{2}}(\psi(x)) d x$. It is also known that (Bashirov et al., 2008), if $\psi$ is positive and 
Riemann integrable on $\left[v_{1}, v_{2}\right]$, then $\psi$ is multiplicative integrable on $\left[v_{1}, v_{2}\right]$ and

$$
\int_{v_{1}}^{v_{2}}(\psi(x))^{d x}=e^{\int_{v_{1}}^{v_{2}} \ln (\psi(x)) d x} .
$$

Bashirov et al. (2008) showed that multiplicative integral has the following results and notations:

1. $\int_{v_{1}}^{v_{2}}\left((\psi(x))^{p}\right)^{d x}=\int_{v_{1}}^{v_{2}}\left((\psi(x))^{d x}\right)^{p}$,

2. $\int_{v_{1}}^{v_{2}}(\psi(x) g(x))^{d x}=$

$\int_{v_{1}}^{v_{2}}(\psi(x))^{d x} \cdot \int_{v_{1}}^{v_{2}}(\phi(x))^{d x}$,

3. $\int_{v_{1}}^{v_{2}}\left(\frac{\psi(x)}{\phi(x)}\right)^{d x}=\frac{\int_{v_{1}}^{v_{2}}(\psi(x))^{d x}}{\int_{v_{1}}^{v_{2}}(\phi(x))^{d x}}$

4. $\int_{v_{1}}^{v_{2}}(\psi(x))^{d x}=\int_{v_{1}}^{\mu}(\psi(x))^{d x} \cdot \int_{\mu}^{v_{2}}(\psi(x))^{d x}$, $v_{1} \leq \mu \leq v_{2}$,

5. $\int_{v_{1}}^{v_{1}}(\psi(x))^{d x}=1$,

6. $\int_{v_{1}}^{v_{2}}(\psi(x))^{d x}=\left(\int_{v_{2}}^{v_{1}}(\psi(x))^{d x}\right)^{-1}$

\section{Main Results}

In this section we obtain some Hermite-Hadamard type integral inequalities in the setting of multiplicative calculus for multiplicatively $P$ functions and convex functions.

Theorem 2.1. Let $\psi$ be a positive and multiplicatively $P$-function on $\left[v_{1}, v_{2}\right]$. Then the following inequalities hold:

$$
\begin{aligned}
\psi\left(\frac{v_{1}+v_{2}}{2}\right) \leq\left(\int_{v_{1}}^{v_{2}}(\psi(x))^{d x}\right)^{\frac{2}{v_{2}-v_{1}}} & \leq\left[\psi\left(v_{1}\right) \psi\left(v_{2}\right)\right]^{2}
\end{aligned}
$$

Proof. If $\psi$ is a positive and multiplicatively $P$ function, then we have

$$
\begin{aligned}
& \ln \psi\left(\frac{v_{1}+v_{2}}{2}\right) \\
& =\ln \left(\psi\left(\frac{(1-\lambda) v_{1}+\lambda v_{2}+\lambda v_{1}+(1-\lambda) v_{2}}{2}\right)\right) \\
& =\ln \left(\psi\left(\frac{(1-\lambda) v_{1}+\lambda v_{2}}{2}+\frac{\lambda v_{1}+(1-\lambda) v_{2}}{2}\right)\right)
\end{aligned}
$$

$$
\begin{gathered}
\leq \ln \left(( \psi ( ( 1 - \lambda ) v _ { 1 } + \lambda v _ { 2 } ) ) \cdot \left(\psi \left(\lambda v_{1}\right.\right.\right. \\
\left.\left.\left.+(1-\lambda) v_{2}\right)\right)\right) \\
=\ln \left(\psi\left((1-\lambda) v_{1}+\lambda v_{2}\right)\right) \\
+\ln \left(\psi\left(\lambda v_{1}+(1-\lambda) v_{2}\right)\right)
\end{gathered}
$$

Integrating the above inequality with respect to $\lambda$ on $[0,1]$, we get

$$
\begin{aligned}
& \ln \psi\left(\frac{v_{1}+v_{2}}{2}\right) \\
& \leq \int_{0}^{1} \ln \left(\psi\left((1-\lambda) v_{1}+\lambda v_{2}\right)\right) d \lambda \\
& +\int_{0}^{1} \ln \left(\psi\left(\lambda v_{1}+(1-\lambda) v_{2}\right)\right) d \lambda \\
& =\left[\frac{1}{v_{2}-v_{1}} \int_{v_{1}}^{v_{2}} \ln (\psi(x)) d x\right. \\
& \left.+\frac{1}{v_{1}-v_{2}} \int_{v_{2}}^{v_{1}} \ln (\psi(x)) d x\right] \\
& =\left[\frac{1}{v_{2}-v_{1}} \int_{v_{1}}^{v_{2}} \ln (\psi(x)) d x\right. \\
& \left.+\frac{1}{v_{2}-v_{1}} \int_{v_{1}}^{v_{2}} \ln (\psi(x)) d x\right] \\
& =\frac{2}{v_{2}-v_{1}} \int_{v_{1}}^{v_{2}} \ln (\psi(x)) d x \text {. }
\end{aligned}
$$

Hence, we have

$$
\begin{aligned}
\psi\left(\frac{v_{1}+v_{2}}{2}\right) \leq & e^{\left(\frac{2}{v_{2}-v_{1}} \int_{v_{1}}^{v_{2}} \ln (\psi(x)) d x\right)} \\
& =\left(\int_{v_{1}}^{v_{2}}(\psi(x))^{d x}\right)^{\frac{2}{v_{2}-v_{1}}} .
\end{aligned}
$$

Thus, we obtain

$$
\psi\left(\frac{v_{1}+v_{2}}{2}\right) \leq\left(\int_{v_{1}}^{v_{2}}(\psi(x))^{d x}\right)^{\frac{2}{v_{2}-v_{1}}}
$$

which completes the proof of the first inequality in (1). Now consider the second inequality in (1).

$$
\begin{aligned}
\left(\int_{v_{1}}^{v_{2}}(\psi(x))^{d x}\right)^{\frac{1}{v_{2}-v_{1}}} & =\left(e^{\left.\left(\int_{v_{1}}^{v_{2}} \ln (\psi(x)) d x\right)\right)^{\frac{1}{v_{2}-v_{1}}}}\right. \\
& =e^{\frac{1}{v_{2}-v_{1}}\left(\int_{v_{1}}^{v_{2}} \ln (\psi(x)) d x\right)} \\
& =e^{\left(\int_{0}^{1} \ln \left(\psi\left(v_{1}+\lambda\left(v_{2}-v_{1}\right)\right)\right) d \lambda\right)} \\
& \leq e^{\left(\int_{0}^{1} \ln \left(\psi\left(v_{1}\right) \psi\left(v_{2}\right)\right) d \lambda\right)} \\
& =e^{\left(\ln \left(\psi\left(v_{1}\right) \psi\left(v_{2}\right)\right)^{\int_{0}^{1} d \lambda}\right)} \\
& =\psi\left(v_{1}\right) \psi\left(v_{2}\right) .
\end{aligned}
$$


Hence, we get

$\left(\int_{v_{1}}^{v_{2}}(\psi(x))^{d x}\right)^{\frac{2}{v_{2}-v_{1}}} \leq\left[\psi\left(v_{1}\right) \psi\left(v_{2}\right)\right]^{2}$.

Combining the inequalities (2) and (3) gives the inequality (1).

Corollary 2.2. Let $\psi$ and $\phi$ be positive and multiplicatively $P$-functions on $\left[v_{1}, v_{2}\right]$. Then the following inequalities hold:

$\psi\left(\frac{v_{1}+v_{2}}{2}\right) \phi\left(\frac{v_{1}+v_{2}}{2}\right)$

$\leq\left(\int_{v_{1}}^{v_{2}}(\psi(x))^{d x} \cdot \int_{v_{1}}^{v_{2}}(\phi(x))^{d x}\right)^{\frac{2}{v_{2}-v_{1}}}$

$\leq\left[\left(\psi\left(v_{1}\right) \psi\left(v_{2}\right)\right) \cdot\left(\phi\left(v_{1}\right) \phi\left(v_{2}\right)\right)\right]^{2}$.

Proof. Since $\psi$ and $\phi$ are positive and multiplicatively $P$-functions, then $\psi \phi$ is a multiplicatively $P$-function. Thus if we apply Theorem 2.1 to the function $\psi \phi$, then we obtain the desired result.

Corollary 2.3. Let $\psi$ and $\phi$ be positive and multiplicatively $P$-functions on $\left[v_{1}, v_{2}\right]$. Then the following inequalities hold:

$$
\begin{aligned}
\frac{\psi\left(\frac{v_{1}+v_{2}}{2}\right)}{\phi\left(\frac{v_{1}+v_{2}}{2}\right)} & \leq\left(\frac{\int_{v_{1}}^{v_{2}}(\psi(x))^{d x}}{\int_{v_{1}}^{v_{2}}(\phi(x))^{d x}}\right)^{\frac{2}{v_{2}-v_{1}}} \\
& \leq\left[\frac{\psi\left(v_{1}\right) \psi\left(v_{2}\right)}{\phi\left(v_{1}\right) \phi\left(v_{2}\right)}\right]^{2}
\end{aligned}
$$

Proof. Since $\psi$ and $\phi$ are positive and multiplicatively $P$-functions, then $\frac{\psi}{\phi}$ is a multiplicatively $P$-function. Thus if we apply Theorem 2.1 to the function $\frac{\psi}{\phi}$, then we obtain the desired result.

Theorem 2.4. Let the positive functions $\psi, \phi$ be convex and multiplicatively $P$-functions, respectively. Then, we have

$$
\begin{aligned}
\left(\frac{\int_{v_{1}}^{v_{2}}(\psi(x))^{d x}}{\int_{v_{1}}^{v_{2}}(\phi(x))^{d x}}\right)^{\frac{1}{v_{2}-v_{1}}} & \left(\frac{\left(\psi\left(v_{2}\right)\right)^{\psi\left(v_{2}\right)}}{\left(\psi\left(v_{1}\right)\right)^{\psi\left(v_{1}\right)}}\right)^{\frac{1}{\psi\left(v_{2}\right)-\psi\left(v_{1}\right)}} \\
& \leq \frac{\left(\phi\left(v_{1}\right) \phi\left(v_{2}\right)\right)}{}
\end{aligned}
$$

Proof. Note that

$$
\begin{aligned}
& \left(\frac{\int_{v_{1}}^{v_{2}}(\psi(x))^{d x}}{\int_{v_{1}}^{v_{2}}(\phi(x))^{d x}}\right)^{\frac{1}{v_{2}-v_{1}}} \\
& =\left(\frac{e^{\int_{v_{1}}^{v_{2}} \ln (\psi(x)) d x}}{e^{\int_{v_{1}}^{v_{2}} \ln (\phi(x)) d x}}\right)^{\frac{1}{v_{2}-v_{1}}} \\
& =\left(e^{\int_{v_{1}}^{v_{2}} \ln (\psi(x)) d x-\int_{v_{1}}^{v_{2}} \ln (\phi(x)) d x}\right)^{\frac{1}{v_{2}-v_{1}}} \\
& =e^{\int_{0}^{1} \ln \left(\psi\left(v_{1}+\lambda\left(v_{2}-v_{1}\right)\right)\right) d \lambda-\int_{0}^{1} \ln \left(\phi\left(v_{1}+\lambda\left(v_{2}-v_{1}\right)\right)\right) d \lambda} \\
& \leq \frac{e^{\int_{0}^{1} \ln \left(\psi\left(v_{1}\right)+\lambda\left(\psi\left(v_{2}\right)-\psi\left(v_{1}\right)\right)\right) d \lambda}}{e^{\int_{0}^{1} \ln \left(\phi\left(v_{1}\right) \phi\left(v_{2}\right)\right) d \lambda}} \\
& =\frac{e^{(n-1}\left(\frac{\left(\psi\left(v_{2}\right)\right)^{\psi\left(v_{2}\right)}}{\left(\psi\left(v_{1}\right)\right)^{\psi\left(v_{1}\right)}}\right)^{\frac{1}{\psi\left(v_{2}\right)-\psi\left(v_{1}\right)}}}{e^{\ln \left(\phi\left(v_{1}\right) \phi\left(v_{2}\right)\right)^{\int_{0}^{1} d \lambda}}} \\
& =\frac{\left(\frac{\left(\psi\left(v_{2}\right)\right)^{\psi\left(v_{2}\right)}}{\left(\psi\left(v_{1}\right)\right)^{\psi\left(v_{1}\right)}}\right)^{\frac{\psi}{\psi\left(v_{2}\right)-\psi\left(v_{1}\right)}}}{e \cdot\left(\phi\left(v_{1}\right) \phi\left(v_{2}\right)\right)}
\end{aligned}
$$

Thus, we have

$$
\begin{aligned}
& \left(\frac{\int_{v_{1}}^{v_{2}}(\psi(x))^{d x}}{\int_{v_{1}}^{v_{2}}(\phi(x))^{d x}}\right)^{\frac{1}{v_{2}-v_{1}}} \\
& \leq \frac{\left(\frac{\left(\psi\left(v_{2}\right)\right)^{\psi\left(v_{2}\right)}}{\left(\psi\left(v_{1}\right)\right)^{\psi\left(v_{1}\right)}}\right)^{\frac{1}{\psi\left(v_{2}\right)-\psi\left(v_{1}\right)}}}{e \cdot\left(\phi\left(v_{1}\right) \phi\left(v_{2}\right)\right)},
\end{aligned}
$$

which completes the proof.

Theorem 2.5. Let the positive functions $\psi, \phi$ be multiplicatively $P$-function and convex function, respectively. Then, we have 


$$
\begin{aligned}
\left(\frac{\int_{v_{1}}^{v_{2}}(\psi(x))^{d x}}{\int_{v_{1}}^{v_{2}}(\phi(x))^{d x}}\right)^{\frac{1}{v_{2}-v_{1}}} & \leq \frac{e \cdot\left(\psi\left(v_{1}\right) \psi\left(v_{2}\right)\right)}{\left(\frac{\left(\phi\left(v_{2}\right)\right)^{\phi\left(v_{2}\right)}}{\left(\phi\left(v_{1}\right)\right)^{\phi\left(v_{1}\right)}}\right)^{\frac{1}{\phi\left(v_{2}\right)-\phi\left(v_{1}\right)}}}
\end{aligned}
$$

Proof. Note that

$$
\begin{aligned}
& \left(\frac{\int_{v_{1}}^{v_{2}}(\psi(x))^{d x}}{\int_{v_{1}}^{v_{2}}(\phi(x))^{d x}}\right)^{\frac{1}{v_{2}-v_{1}}} \\
& =\left(\frac{e^{\int_{v_{1}}^{v_{2}} \ln (\psi(x)) d x}}{\left.e^{\int_{v_{1}}^{v_{2}} \ln (\phi(x)) d x}\right)^{\frac{1}{v_{2}-v_{1}}}}\right. \\
& =\left(e^{\int_{v_{1}}^{v_{2}} \ln (\psi(x)) d x-\int_{v_{1}}^{v_{2}} \ln (\phi(x)) d x}\right)^{\frac{1}{v_{2}-v_{1}}} \\
& =\frac{e^{\int_{0}^{1} \ln \left(\psi\left(v_{1}+\lambda\left(v_{2}-v_{1}\right)\right)\right) d \lambda-\int_{0}^{1} \ln \left(\phi\left(v_{1}+\lambda\left(v_{2}-v_{1}\right)\right)\right) d \lambda}}{e^{\int_{0}^{1} \ln \left(\phi\left(v_{1}\right)+\lambda\left(\phi\left(v_{2}\right)-\phi\left(v_{1}\right)\right)\right) d \lambda}} \\
& \left.=\frac{e^{\int_{0}^{1} \ln \left(\psi\left(v_{1}\right) \psi\left(v_{2}\right)\right) d \lambda}}{\ln \left(\left(\frac{\left(\phi\left(v_{2}\right)\right)^{\phi\left(v_{2}\right)}}{\left(\phi\left(v_{1}\right)\right)^{\phi\left(v_{1}\right)}}\right)^{\phi\left(v_{2}\right)-\phi\left(v_{1}\right)}\right.}\right)-1 \\
& =\frac{e \cdot\left(\psi\left(v_{1}\right) \psi\left(v_{2}\right)\right)}{\left(\frac{\left(\phi\left(v_{2}\right)\right)^{\phi\left(v_{2}\right)}}{\left(\phi\left(v_{1}\right)\right)^{\phi\left(v_{1}\right)}}\right)^{\frac{1}{\phi\left(v_{2}\right)-\phi\left(v_{1}\right)}}} .
\end{aligned}
$$

Hence, we have

$$
\begin{aligned}
\left(\frac{\int_{v_{1}}^{v_{2}}(\psi(x))^{d x}}{\int_{v_{1}}^{v_{2}}(\phi(x))^{d x}}\right)^{\frac{1}{v_{2}-v_{1}}} & \leq \frac{e \cdot\left(\psi\left(v_{1}\right) \psi\left(v_{2}\right)\right)}{\left(\frac{\left(\phi\left(v_{2}\right)\right)^{\phi\left(v_{2}\right)}}{\left(\phi\left(v_{1}\right)\right)^{\phi\left(v_{1}\right)}}\right)^{\frac{1}{\phi\left(v_{2}\right)-\phi\left(v_{1}\right)}}}
\end{aligned}
$$

which is the desired result.

Theorem 2.6. Let the positive functions $\psi, \phi$ be convex and multiplicatively $P$-functions, respectively. Then, we have

$$
\begin{aligned}
& \left(\int_{v_{1}}^{v_{2}}(\psi(x))^{d x} \cdot \int_{v_{1}}^{v_{2}}(g(x))^{d x}\right)^{\frac{1}{v_{2}-v_{1}}} \\
& \leq \frac{\left(\frac{\left(\psi\left(v_{2}\right)\right)^{\psi\left(v_{2}\right)}}{\left(\psi\left(v_{1}\right)\right)^{\psi\left(v_{1}\right)}}\right)^{\frac{1}{\psi\left(v_{2}\right)-\psi\left(v_{1}\right)}} \cdot\left(\phi\left(v_{1}\right) \phi\left(v_{2}\right)\right)}{e} .
\end{aligned}
$$

Proof. Note that

$$
\begin{aligned}
& \left(\int_{v_{1}}^{v_{2}}(\psi(x))^{d x} \cdot \int_{v_{1}}^{v_{2}}(\phi(x))^{d x}\right)^{\frac{1}{v_{2}-v_{1}}} \\
& =\left(e^{\int_{v_{1}}^{v_{2}} \ln (\psi(x)) d x+\int_{v_{1}}^{v_{2}} \ln (\phi(x)) d x}\right)^{\frac{1}{v_{2}-v_{1}}} \\
& =\left(e^{\int_{v_{1}}^{v_{2}} \ln (\psi(x)) d x} \cdot e^{\int_{v_{1}}^{v_{2}} \ln (\phi(x)) d x}\right)^{\frac{1}{v_{2}-v_{1}}} \\
& =\left(e^{\left(v_{2}-v_{1}\right) \int_{0}^{1} \ln \left(\psi\left(v_{1}+\lambda\left(v_{2}-v_{1}\right)\right)\right) d \lambda}\right)^{\frac{1}{v_{2}-v_{1}}} \\
& \times\left(e^{\left(v_{2}-v_{1}\right)\left(\int_{0}^{1} \ln \left(\phi\left(v_{1}+\lambda\left(v_{2}-v_{1}\right)\right)\right) d \lambda\right)}\right)^{\frac{1}{v_{2}-v_{1}}} \\
& =e^{\int_{0}^{1} \ln \left(\psi\left(v_{1}+\lambda\left(v_{2}-v_{1}\right)\right)\right) d \lambda} \cdot e^{\int_{0}^{1} \ln \left(\phi\left(v_{1}+\lambda\left(v_{2}-v_{1}\right)\right)\right) d \lambda} \\
& \leq e^{\int_{0}^{1} \ln \left(\psi\left(v_{1}\right)+\lambda\left(\psi\left(v_{2}\right)-\psi\left(v_{1}\right)\right)\right) d \lambda} \\
& \times e^{\int_{0}^{1} \ln \left(\phi\left(v_{1}\right) \phi\left(v_{2}\right)\right) d \lambda} \\
& =e^{\ln \left(\left(\frac{\left(\psi\left(v_{2}\right)\right)^{\psi\left(v_{2}\right)}}{\left(\psi\left(v_{1}\right)\right)^{\psi\left(v_{1}\right)}}\right)^{\frac{1}{\psi\left(v_{2}\right)-\psi\left(v_{1}\right)}}\right)-1} \\
& \times e^{\ln \left(\phi\left(v_{1}\right) \phi\left(v_{2}\right)\right)^{\int_{0}^{1} d \lambda}} \\
& =\frac{\left(\frac{\left(\psi\left(v_{2}\right)\right)^{\psi\left(v_{2}\right)}}{\left(\psi\left(v_{1}\right)\right)^{\psi\left(v_{1}\right)}}\right)^{\frac{1}{\psi\left(v_{2}\right)-\psi\left(v_{1}\right)}} \cdot\left(\phi\left(v_{1}\right) \phi\left(v_{2}\right)\right)}{e} .
\end{aligned}
$$

Thus,

$$
\begin{aligned}
& \left(\int_{v_{1}}^{v_{2}}(\psi(x))^{d x} \cdot \int_{v_{1}}^{v_{2}}(\phi(x))^{d x}\right)^{\frac{1}{v_{2}-v_{1}}} \\
& \leq \frac{\left(\frac{\left(\psi\left(v_{2}\right)\right)^{\psi\left(v_{2}\right)}}{\left(\psi\left(v_{1}\right)\right)^{\psi\left(v_{1}\right)}}\right)^{\frac{1}{\psi\left(v_{2}\right)-\psi\left(v_{1}\right)}} \cdot\left(\phi\left(v_{1}\right) \phi\left(v_{2}\right)\right)}{e} .
\end{aligned}
$$

This completes the proof.

\section{Acknowledgments}

This research article is supported by Kurklareli University Scientific Research Projects 
Coordination Unit. Project Number: KLUBAP191.

\section{References}

Ali, M.A., Abbas, M., Zhang, Z., Sial, I.B. ve Arif, R., 2019. On Integral Inequalities for Product and Quotient of Two Multiplicatively Convex Functions. Asian Research Journal of Mathematics, 12(3), 1-11.

Bashirov, A.E., Kurpınar, E.M. ve Özyapıcı, A., 2008. Multiplicative Calculus and Applications. Journal of Mathematical Analysis and Applications, 337(1), 36-48.

Budak, H., Tunç, T. ve Sarıkaya, M.Z., 2019. On Hermite-Hadamard Type Inequalities for FConvex Function. Miskolc Mathematical Notes, 20(1), 169-191.

Dragomir, S.S. ve Pearce, C.E.M., 1998. Quasi-Convex Functions and Hermite-Hadamard's Inequality. Bulletin of the Australian Mathematical Society, 57, 377-385.

Dragomir, S.S. ve Pearce, C.E.M., 2000. Selected Topics on Hermite-Hadamard Inequalities and Applications, RGMIA Monographs, Victoria University of Technology, Victoria, 357p.

Dragomir, S.S., Pecaric, J. ve Persson, L.E., 1995. Some Inequalities of Hadamard Type. Soochow Journal of Mathematics, 21(3), 335-341.

Kadakal, H., 2018. Multiplicatively P-Functions and Some New Inequalities. New Trends in Mathematical Sciences, 6(4), 111-118.

Kadakal, H., 2019. Some Integral Inequalities for Multiplicatively Geometrically P-Functions. An International Journal of Optimization and Control: Theories \& Applications, 9(2), 216222.
Kadakal, M., 2019. Hermite-Hadamard and Simpson Type Inequalities for Multiplicatively Harmonically P-Functions. Sigma Journal of Engineering and Natural Sciences, 37(4), 13111320 .

Maden, S., Demirel, A.K., Turhan, S. ve İşcan, İ., 2018. Some Integral Inequalities for the New Convex Functions. Sigma Journal of Engineering and Natural Sciences, 9(3), 319326.

Özcan, S., 2019. Some Integral Inequalities for Harmonically $(\alpha, s)$-Convex Functions. Journal of Function Spaces, 2019, Article ID 2394021, $1-8$.

Özcan, S. ve İşcan, İ., 2019. Some New HermiteHadamard Type Inequalities for s-Convex Functions and Their Applications. Journal of Inequalities and Applications, 2019(201), 1-11.

Özdemir, M.E., Önalan, H.K. ve Ardıç, M.A., 2015. Hermite-Hadamard Type Inequalities for (h$(\alpha, \mathrm{m}))$-Convex Functions. Journal of Concrete \& Applicable Mathematics, 13, 96-107.

Pečarić, J.E., Proschan, F. ve Tong, Y.L., 1992. Convex Functions, Partial Orderings and Statistical Applications, Academic Press, Boston, 467p.

Sarıkaya, M.Z., Akkurt, A., Budak, H., Yıldırım, M.E. ve Yıldırım, H., 2019. Hermite-Hadamard's Inequalities for Conformable Fractional Integrals. An International Journal of Optimization and Control: Theories \& Applications, 9(1), 49-59.

Set, E., İşcan, İ., Sarıkaya, M.Z. ve Özdemir, M.E., 2015. On New Inequalities of HermiteHadamard-Fejer Type for Convex Functions via Fractional Integrals. Applied Mathematics and Computation, 259, 875-881. 